\title{
Research on the Evolution Method of Cluster Supply Chain Network Based on Computational Experiment
}

\author{
http://dx.doi.org/10.3991/ijoe.v9iS4.2661
}

\author{
Ji-yu An, Yu-qi Zhai, Xiao Xue, Hui-juan Zhang and Shan-shan Yang \\ Henan Polytechnic University, Jiaozuo, China
}

\begin{abstract}
The definition of cluster supply chain (CSC) is proposed to help small and medium-sized enterprises (SMEs) realize "ant colony effect" and gain more profits. Considering CSC is a complex adaptive dynamic system, it is still in the initial stage and the problem of evolution is difficult to solve by static method. This paper builds a twostage supply chain evolution model based on computational experiment and generates Agent models. The evolution model is extended to three-stage supply chain model in simulation to research the influence factors of network evolution and predict the effectiveness of service support system, supporting the development of CSC. Preliminary results show that the evolution model can provide the reference for the development of CSC.
\end{abstract}

Index Terms-CSC, Computational Experiment, Evolution Model, Coordination Procurement

\section{INTRODUCTION}

With the advent of the era of economic globalization, it brings about tremendous development opportunities for enterprises and also causes them to face unprecedented challenges at the same time. CSC is proposed as a new management pattern to cope with the increasing competition [1]. With the help of CSC, it gives full play to the strengths of industrial cluster and supply chain and realizes the advantages of clear division of industry chain, self-organization, etc., helping SMEs realize the purpose of "Small broad".

Enterprises form the scale effect, which can reduce costs and generate profits through coordination based on the theory of CSC. The types of coordination include: coordination procurement [2], coordination manufacturing, coordination logistics, etc. Procurement provides material support for enterprises as the first step of production, which plays a key role in the operation of enterprises.

The research of CSC is still in its initial stage [3]: on one hand, the research about CSC is more from economics and management at the current stage. On the other hand it lacks the support of technology. CSC is a complex evolving system which is composed of a large number of enterprises with the goal of maximizing their potential profit through interactions, and the evolution path has variability. So models which are built by conventional mathematical methods can not abstract CSC well, for they are restricted by many kinds of factors. The method of computational experiment can simulate the interactions between enterprises, whole emergence and evolution process of CSC, so CSC can be researched thoroughly.

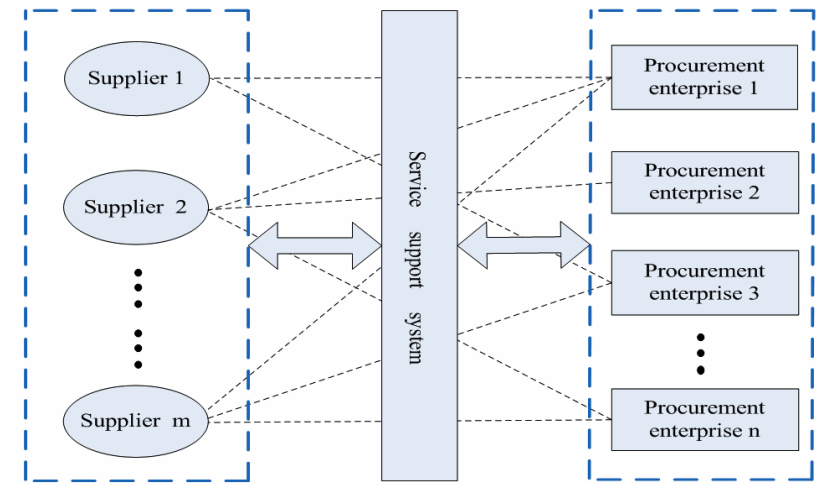

Figure 1. Coordination procurement with a service support system

Adopting computational experiment can not only analyze the phenomenon which has happened but also is happening or will happen and explain the causes, influences and rules of the phenomenon. This paper takes the coordination procurement of CSC as background, uses computational experiment to build an evolution model through simulating the enterprise behaviors and interactions. It adopts the technologies of Agent [4-5] to build models in the cluster, emerging overall behaviors and evolution of CSC network from the bottom up based on the interaction between enterprises.

\section{RELATED WORK}

CSC is a really complex adaptive system which can be demonstrated in the following aspects. First, it is composed of multi-interest enterprises, which changes dynamically. Second, CSC has locality. Third, enterprises in the cluster have autonomy and intelligence.

Information technology analyzes supply chain by getting, transferring and processing data, which helps managers plan tasks at a higher level. Information technology is combined with economic management technology to support the coordination of CSC, promoting the development of CSC [6]. So, service support system is proposed to compromise the bound between business models and information technology, as Fig. 1 shows.

\section{EVOLUTION MODEL OF CSC NETWORK}

\section{A. Model Definition and Constrains}

Enterprises procure, manufacture and sell according to their own information, calculating their profits and enterprises take which as a measure to view their own behav- 
iors and the state of business. In the cluster, retailers face consumer market and suppliers face raw material market. Suppose all procurement enterprises procure the same products, suppliers provide the required products and downstream enterprises procure from their upstream enterprises in the supply chain. The procurement relationships are one-to-one or many-to-one.

\section{B. The Construction of the Coordination Procurement Models}

\section{1) The model of procurement enterprise Agents}

Individual model: The objective properties of procurement enterprise Agents change with time, including: scale (cScale), growth rate(cRate), procurement ideal price(idealpr), procurement batch(cBatch), product selling price(cPrice), product selling number(cNum), identity number(cID), and profits(cProf).

1. The scale of procurement enterprise Agents changes with time

According to logistic model, the scale of enterprise Agents grows according to the growth rate of the current time and the growth rate of enterprise Agents at time t can be calculated as:

$$
d x_{i}(t) / d t=r_{i}(t) x_{i}(t)\left(1-x_{i}(t) / k_{i}\right)
$$

Where $x_{i}(t)$ denotes the scale of enterprise Agents at time t, $r_{i}(t)$ denotes the intrinsic growth rate of enterprises at time $\mathrm{t}$ and $k_{i}$ is the maximum enterprise scale, all of which are achieved only rely on limited resources.

Coordination in this case, the growth rate of enterprises can be calculated as:

$$
d x_{i}(t) / d t=r_{i}(t) x_{i}(t)\left(1-x_{i}(t) / k_{i}+\text { coopAdd }\right)
$$

The growth rate of enterprise Agents is influenced by horizontal coordination (enterprises coordinate with homogeneous enterprises) effect. And coopAdd denotes the horizontal coordination effect with the value is $\sum a_{i j} x_{j}(t) / k_{j}, a_{i j}$ is the coordination effect coefficient which means the incidence of coordination enterprise Agent $\mathrm{j}$ to procurement enterprise Agent $\mathrm{i}$, and $-1<$ $a_{i j}<1$.

Contrasting (1) and (2), it is easy to find that enterprises can develop rapidly by horizontal coordination.

2. The profits of procurement enterprise Agents change with time

The profits of procurement enterprise Agents can be calculated as:

$$
c \operatorname{Prof}(t)=\operatorname{in}(t)-c o(t)
$$

Where in $(\mathrm{t})$ denotes the earnings of enterprises at time $t$, the value is $i n(t)=c \operatorname{Pr}$ ice ${ }^{*} c N u m$. And co(t) denotes the costs of procurement enterprises at time $t$, the value is: $c o(t)=\operatorname{pur}_{c o}(t)+\log _{c o}+s t o_{c o}+c o o p$.And $\operatorname{pur}_{\mathrm{co}}(\mathrm{t})$ denotes the procurement costs of procurement enterprises, $\log _{\mathrm{co}}(\mathrm{t})$ is the storage costs, which are all non-linear proportional to beach, $\operatorname{coop}_{\mathrm{co}}(\mathrm{t})$ is the coordination management costs, it includes the fixed costs of coordination behavior and a percentage of every coordination earnings, which can be calculated:

$$
\text { pri_alli }=(\text { idealpr }- \text { s Price }) * \text { cBatch } * \alpha .
$$

In the Eq. (4), $\alpha$ is the fixed rate. Suppose the current sale price and number of procurement enterprises do not change, and then the profits of the procurement enterprise Agents are driven by costs.

Coordination model: Enterprise Agents are not stationary, passively waiting, but active enough to change market environment. They adjust behaviors according to the market environment to achieve the desired goal. Under the market economy, enterprises in the cluster aim for maximizing profits. Therefore, enterprise profits are the major factor that stimulates enterprise behaviors to change, and the changes of behaviors push the development of enterprises. Coordination can form scale effect to increase enterprise profits based on the theory of CSC. The coordination behaviors of procurement enterprise Agents include: 1. procurement enterprise Agents select the best supplier Agent; 2. procurement enterprise Agents select other homogeneous enterprise Agents of different supply chains to coordinate; 3. procurement enterprise Agents coordinate with a service support system Agent.

1. Procurement enterprise Agents select the best supplier Agent

Procurement enterprise Agents select the best supplier Agent according to their own demands, a variety of factors need considering include: cooperative successful rate, product price, quality, delivery capability, after-sale service, etc. The vertical coordination (enterprises coordinate with heterogeneous enterprises) rule means procurement enterprise Agents select the best supplier Agent, falling into two categories: the vertical coordination rule of SMEs and the vertical coordination rule of large-scale enterprises. SMEs view supplier Agent ID sequentially in a relatively small range due to their limited abilities. The large-scale enterprises can select the best supplier in a certain range of selection, the evaluation function is:

$$
R^{\prime}=\alpha^{*} s \operatorname{Pr} i c e+\beta * s Q u a l i t y+\delta * s \text { Ability }
$$

$$
\text { Where } 0 \leq \alpha, \beta, \delta \leq 1 \text { and } \alpha+\beta+\delta=1 \text {. }
$$

The process that procurement enterprise Agents coordinate with supplier Agents is depicted as follows. Procurement enterprise Agents view supplier Agents sequentially according to their own rules. Cooperative successful rate reflects cooperative relationship, credit and capabilities of supplier Agents, which has a great influence on the next cooperation decision-marking of procurement enterprises, so it needs to be considered first. If cooperative successful rate is greater than fifty, then procurement enterprise Agents consider the product price of the supplier Agent. Procurement enterprise Agents and the supplier Agent reach a coordination agreement when there are no procurement relationships between them. 
2. Procurement enterprise Agents select other homogeneous enterprise Agents of different supply chains to coordinate

Procurement enterprise Agents select other homogeneous Agents, falling into two categories: one is procurement enterprise Agents find other homogeneous enterprise Agents to coordinate according to the cooperative relationship of a supplier Agent, another is procurement enterprise Agents coordinate with a large-scale procurement enterprise Agent, forming a virtual procurement alliance with the centre of the large-scale procurement enterprise Agent to procure, which evolves based on the first rule.

The process of procurement enterprise Agents select other homogeneous enterprise Agents to coordinate is depicted as follows. Procurement enterprise Agents view the cooperative successful rate of other homogeneous enterprise Agents according to their own rules. If the cooperative successful rate is greater than fifty, then procurement enterprise Agents view the batch. The horizontal coordination relationships will be viewed, if the batch fluctuates in a small range of their batches. Then procurement enterprise Agents build horizontal coordination relationships with the homogeneous enterprise Agent when there are no relationships between them.

3. Procurement enterprise Agents coordinate with a service support system Agent

When a virtual procurement alliance with the centre of a large-scale procurement enterprise Agent appears in the cluster, size of the alliance exceeds a certain size, leading to growth in coordination management costs and reduction in profits. Web service technology with self-contained, self-described, interoperability and modularization develops rapidly in recent years. Web service makes resources distributing in network constitute a virtual computer system and completes tasks through reusing resources and coordination. Therefore, coordination management tasks are separated from large-scale procurement enterprise Agents which becomes a service support system. With this platform, it can reduce the redundant interaction, coordination costs and increase procurement efficiency.

Procurement enterprise Agents interact with the service support system Agent based entirely on their coordination attribute which means procurement enterprise Agents encapsulate their functions as corresponding Web services and interact with the service support system Agent.

\section{2) The model of supplier Agents}

Individual model: The objective properties of supplier Agents change with time, including: cooperation linked list, scale (sScale), growth rate(sRate), procurement batch(sBatch), product selling price(sPrice), product selling number(sNum), identity number(sID), coordination attribute(sAtt) and profits(sProf).

1. The product price of supplier Agents changes with batch

The product price of supplier Agents (sPrice) varies inversely proportional to the batch of the procurement enterprise Agent (cBatch). Within a certain scope, the larger the cBatch is, the lower the sPrice will be. Finally, the product price will maintain at a relatively stable range for cost constraint and other problems.

2. The size of supplier Agents changes with time
The growth rate of supplier Agents in the coordination can be calculated as:

$$
\begin{aligned}
& d x_{i}(t) / d t= \\
& r_{i}(t) x_{i}(t)\left(1-x_{i}(t) / k_{i}+\operatorname{coop} A d d+\text { procAdd }\right)
\end{aligned}
$$

Where procAdd denotes vertical coordination effect that procurement enterprise Agent $\mathrm{p}$ selects the current supplier Agent $\mathrm{i}$ to procure, and the value is $\sum a_{p i} x_{p}(t) / k_{p}$. In the Eq. (6), $a_{p i}$ is the coordination effect coefficient which means the incidence of coordination enterprise Agent $\mathrm{p}$ to supplier Agent $\mathrm{i}$, and $-1<a_{p i}<1$. $x_{p}(t)$ is the scale at time $\mathrm{t}$ and $k_{p}$ is the maximum enterprise scale of coordination enterprise Agent $\mathrm{p}$.

Contrasting the Eq. (1) and Eq. (6), the scale of supplier Agents is under the influence of horizontal and vertical coordination effect at the same time.

3. The profits of supplier Agents change with time

The profits of supplier Agents are similar to the profits of procurement enterprise Agents. Suppose the sale price and number are constant, logistics costs and storage costs are constant too. The profits of supplier Agents are determined by the coordination management costs.

Coordination model: The coordination behaviors of supplier Agents include: 1.supplier Agents select other homogeneous enterprise Agents of different supply chains to coordinate; 2 . supplier Agents coordinate with a service support system Agent. The behaviors of supplier Agents are similar to procurement enterprise Agents.

3) The model of service support system Agents

Individual model: The objective properties of service support system Agent include: cooperation linked list (relationList), identity number (sID) and profits (sProf).

Coordination model: Procurement enterprise Agents commit procurement demands to service support system Agents as the demand side of coordination procurement model. Service support system Agents merge demands from different procurement enterprise Agents and select the best supplier Agent by open tender. The coordination model includes: 1 . tender releasing; 2 . service support system Agents select the best supply Agent.

1. Tender releasing

Service support system Agents split and merge demands from procurement enterprise Agents, generating the corresponding bid documents which release to supplier Agents. The bid document is expressed as a triplet, which can be calculated as:

$$
<\text { VPC }, T, C>\text {. }
$$

VPC means a virtual procurement alliance which is the tender of procurement tasks. $\mathrm{T}$ means the procurement tasks of the virtual procurement alliance which are procured products. $\mathrm{C}$ means constraint conditions that procurement enterprise Agents select the supplier Agent, which can be calculated as a quad:

$$
C=<P, Q, A, S>
$$


$\mathrm{P}$ is the procurement price, $\mathrm{Q}$ is the product quality, $\mathrm{A}$ is the delivery capability and $S$ is after-sale service of the supplier Agent. Service support system Agents bid for procurement tasks and select the best supplier Agent before the deadline.

2. Service support system Agents select the best supplier Agent

Service support systems select the best supplier based on the evaluation results through Eq. (9) which can be calculated as:

$$
R^{\prime}=\alpha^{*} s \operatorname{Pr}+\beta^{*} s Q u+\delta^{*} s A b+\varepsilon^{*} s S e
$$

Where $0 \leq \alpha, \beta, \delta, \varepsilon \leq 1$ and $\alpha+\beta+\delta+\varepsilon=1$. After viewing the evaluation result, service support system Agents give result information back to participating supplier Agents that send successful news to the successful bidders and return reject news to the losing bidders. Vertical coordination relationships will be built if there are no vertical coordination relationships between service support system Agents and the supplier Agent.

\section{MODEL SIMULATION}

\section{A. Model Environment}

The model environment is a virtual space of 800 by 800. A circle represents an enterprise Agent, the number within which is the enterprise Agent's ID and a straight line with a small square represents a coordination relationship between Agents and the square shows the direction of coordination. Suppose supply chains in the cluster are all three-stage supply chains and the space is divided into three corresponding layers: higher space within which are supplier Agents, middle space within which are manufacturer Agents and lower space within which are retailer Agents.

\section{B. The Result of Simulation}

The evolution of CSC network develops four stages according to Agent models, including the first stage: procurement enterprises only procure without coordination, the second stage: procurement enterprises procure firstly and coordinate lately, the third stage: procurement enterprises coordinate firstly and procure lately and the fourth stage: procurement enterprises procure with a service support system.

The first stage: procurement enterprises only procure without coordination. The scale of all enterprise Agents is a random number within a small range in the initial stage. Enterprise Agents select their upstream supplier Agents according to constraint conditions and submit orders to them to procure, forming many straight lines. For retailer Agents and manufacturer Agents, the square on a straight line near the manufacturer Agent means the procurement relationship is initiated by a retailer Agent, as shown in Fig.2.

In this stage, retailer Agents view manufacturer Agents according to ID orderly and select the first manufacturer Agent which price is lower than their own procurement ideal price. There are drawbacks in this stage that the selected manufacturer Agent is not the optimal one.

The second stage: procurement enterprises procure firstly and coordinate lately. Retailer Agents tend to coordinate to reach mutual benefit and update information timely by sharing information with each other. Enterprises in the cluster procure with coordination which means retailer Agents procure from the same manufacturer Agent interact with each other to reduce costs and share market information through increasing procurement batch. A red straight line with two squares represents a horizontal coordination relationship, which means the coordination relationship is initiated by both sides and virtual procurement alliances with large quantity and small scale are formed. With virtual procurement alliances, procurement enterprise Agents can catch the changing market opportunity quickly by sharing information and complementing advantages. Differences in scale of enterprise Agents emerge in this stage. But procurement alliances have obvious drawbacks that they aren't perfect, the scale is still small, as shown in Fig.3.

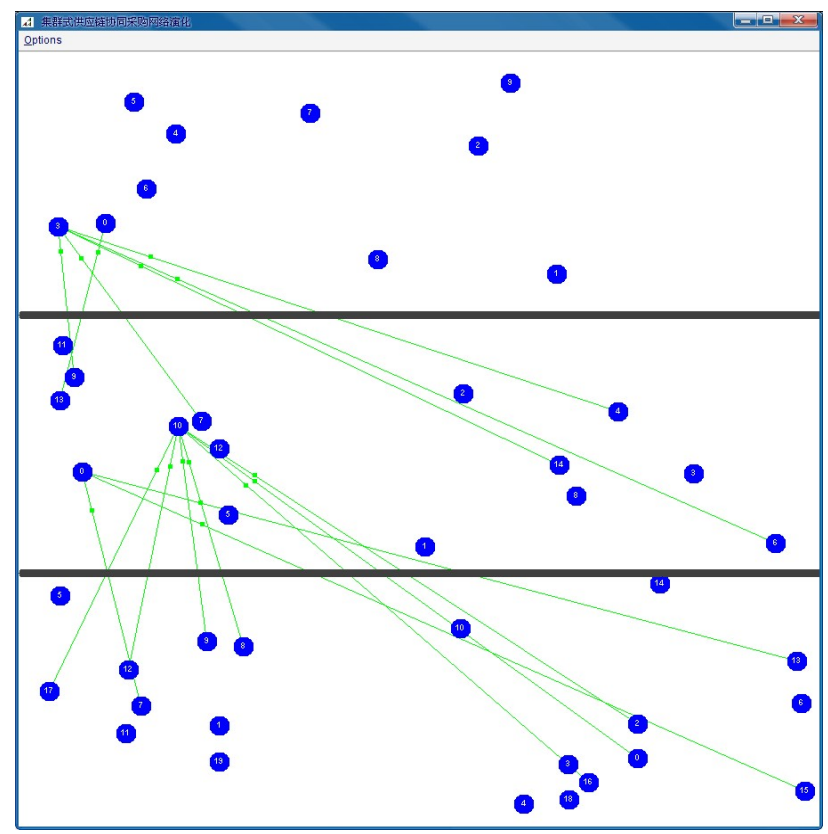

Figure 2. The first stage of the CSC network evolution

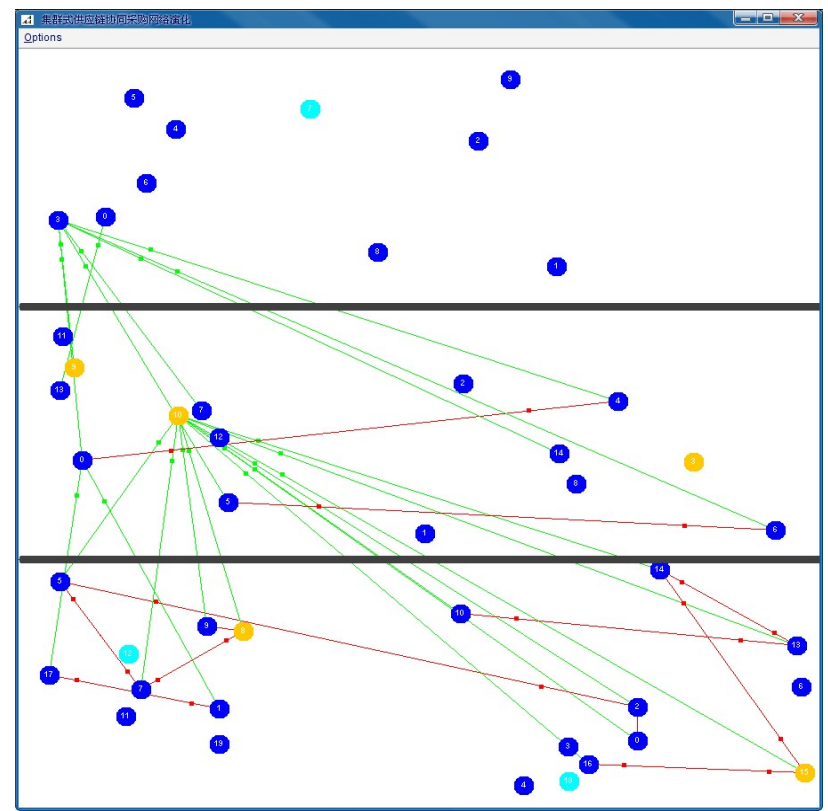

Figure 3. The second stage of the CSC network evolution 


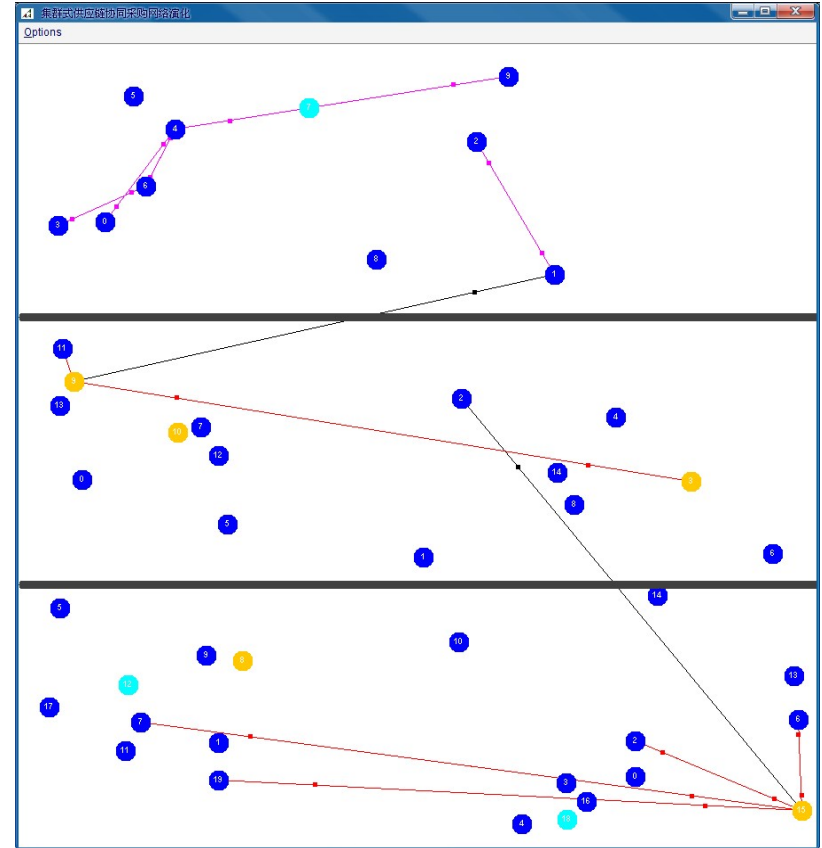

Figure 4. The third stage of the CSC network evolution

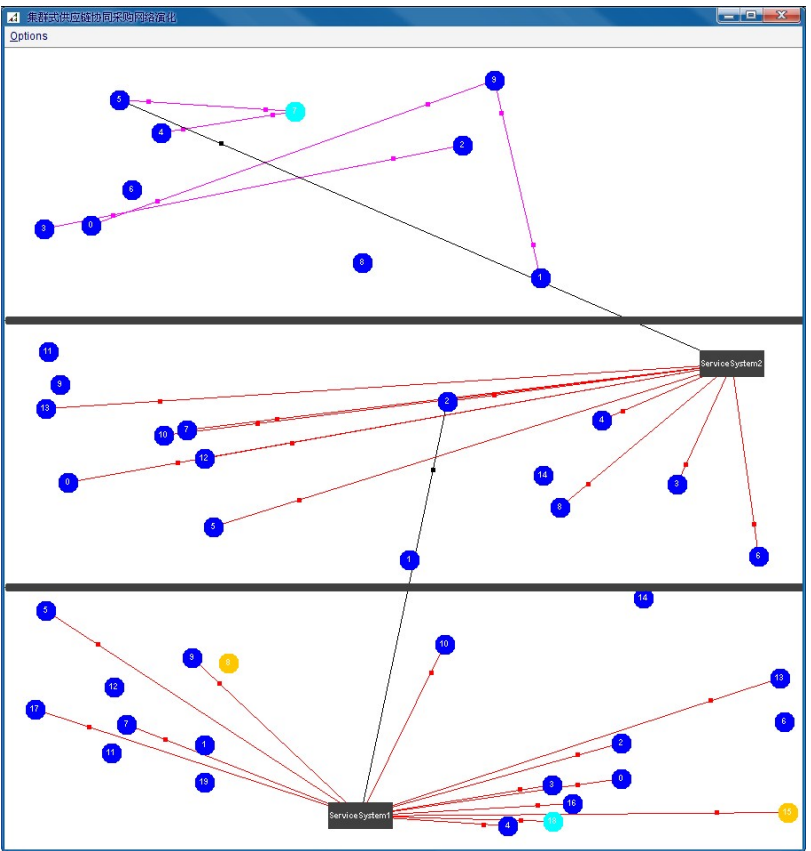

Figure 5. The forth stage of the CSC network evolution

The third stage: procurement enterprises coordinate firstly and procure lately, as shown in Fig.4.

Large-scale enterprise Agents at all levels appear in this stage. Middle and small scale enterprise Agents learn from large-scale enterprise Agents and interact with them to form a virtual procurement alliance, which contributes to seeking the development better and faster. Having large scale, strong capabilities, good reputation and rich information of the industry, large-scale enterprise Agents act as organizers and are the center of virtual procurement alliances. They select the manufacturer Agent and coordinate with it, changing the virtual procurement alliance to the virtual coordination alliance. But for capacity constraints, focal enterprise Agents select manufacturer Agent only in a certain range. But it also has some drawbacks such as the management of virtual coordination alliances is not perfect and the procurement costs need further reduce.

The fourth stage: procurement enterprises procure with a service support system, as is shown in Fig.5.

Service support system Agents separate the functions that integrate orders and select the best manufacturer Agent from focal procurement enterprise Agents. As Fig.5 shows, there is a service support system in every level and most enterprise Agents choose the support system Agent to coordinate with in order to gain more profits and lower procurement price. Service support systems provide platform support which speeds the flow of information, reduces the redundant interaction and procurement costs and unifies the procurement process. It is more systematic and comprehensive in the selection of supplier Agents

\section{CONCLUSIONS}

This paper builds an evolution model based on the bottom-up computational experiment method and uses Agent technology to generate models by extracting behavior mechanism abstractly, finding that every type of the model with different behavior properties and rules makes decisions to cooperate and compete with each other, which promote the evolution of CSC. After analyzing the results of simulation, the interdependence process between enterprises is the evolution process, which contributes to the development of CSC and service support system helps develop CSC. Evolution model still needs to be modified in an iterative matter to optimize it in practical system applications, so as to research and better understand CSC.

\section{ACKNOWLEDGMENT}

Thanks to the supports of National Natural Science Foundation of China (61175066), National Natural Science Foundation of China (60905041) and China Postdoctoral Science Foundation (20110490396).

\section{REFERENCES}

[1] Li Ji-zi, "Study on cluster supply chain and its management," Ph.D. dissertation, Huazhong Agricultural University, Wuhan, China, 2006.

[2] Xue Xiao and Wang yang, "The complexity research for coordination procurement based on cluster supply chain," Journal of Guangxi University (Natural Science Edition), vol.36, pp. 770-780, October 2011.

[3] Xue Xiao and Li Hui-Qin, "Design approach to the service systems of cluster supply chain," Computer Engineering and Science, vol. 32, pp. 161-164, 2010.

[4] Wooldridge $\mathrm{M}$ and Jennings $\mathrm{N}$ R, "Intelligent agent: theory and practice,” The Knowledge Engineering Review, vol. 10, pp. 115152, 1995. http://dx.doi.org/10.1017/S0269888900008122

[5] Jennings N R, Sycara K and Wooldridge M J, “A roadmap of agent research and development", Autonomous Agents and Multi Agent Systems, vol. 1, pp. 7-38, 1998. http://dx.doi.org/10.1023/ A:1010090405266

[6] Xue Xiao, Zhu Peng and Huang Bi-qing, "The Research on agent oriented design approach for service system of cluster supply chain,” Journal of Chinese Computer Systems, vol. 32, pp. 17701777, September 2011. 
SPECIAL FOCUS PAPER

Research on the Evolution Method of Cluster Supply Chain Network Based on Computational Experiment

\section{AUTHORS}

Ji-yu An is with Henan Polytechnic University, Jiaozuo, 454000 China. (e-mail: ajy770406153@163.com).

Yu-qi Zhai is with Henan Polytechnic University, Jiaozuo, 454000 China. (e-mail: zhaiyuqikuro@126.com).

Xiao Xue is with Henan Polytechnic University, Jiaozuo, 454000 China. (e-mail: jzxuexiao@126.com).

This work was supported by the National Science Foundation of China (61175006), National Natural Science Foundation of China (60905041) and China Postdoctoral Science Foundation (20110490396). This article is an extended and modified version of a paper presented at the International Conference on Mechanical Engineering, Automation and Material Science (MEAMS2012), held 22-23 December 2012, Wuhan, China.Manuscrip received 06 April 2013. Received 08 April 2013. Published as resubmitted by the authors 01 May 2013. 